\title{
Results of the CAPSID randomized trial for high-dose convalescent plasma in patients with severe COVID-19
}

\author{
Sixten Körper, ${ }^{1}$ Manfred Weiss, ${ }^{2}$ Daniel Zickler, ${ }^{3}$ Thomas Wiesmann, ${ }^{4}$ Kai Zacharowski, ${ }^{5}$ Victor M. Corman, ${ }^{6}$ Beate Grüner, \\ Lucas Ernst, ${ }^{3}$ Peter Spieth, ${ }^{8}$ Philipp M. Lepper, ${ }^{9}$ Martin Bentz, ${ }^{10}$ Sebastian Zinn, ${ }^{5}$ Gregor Paul, ${ }^{11}$ Johannes Kalbhenn, ${ }^{12}$ \\ Matthias M. Dollinger, ${ }^{13}$ Peter Rosenberger, ${ }^{14}$ Thomas Kirschning, ${ }^{15}$ Thomas Thiele, ${ }^{16}$ Thomas Appl, Benjamin Mayer, ${ }^{17}$ \\ Michael Schmidt, ${ }^{18}$ Christian Drosten, ${ }^{6}$ Hinnerk Wulf, ${ }^{4}$ Jan Matthias Kruse, ${ }^{3}$ Bettina Jungwirth, ${ }^{2}$ Erhard Seifried, ${ }^{18}$ and \\ Hubert Schrezenmeier ${ }^{1}$ for the CAPSID Clinical Trial Group ${ }^{19}$
}

IInstitute for Clinical Transfusion Medicine and Immunogenetics Ulm, German Red Cross Blood Transfusion Service Baden-Württemberg-Hessen and University Hospital Ulm, and Institute of Transfusion Medicine, and '2Department of Anaesthesiology and Intensive Care Medicine, University Hospital Ulm, Ulm University, Ulm, Germany. ${ }^{3}$ Department of Nephrology and Medical Intensive Care, Charité Universitätsmedizin Berlin, corporate member of Free University Berlin, Humboldt-Universität zu Berlin, and Berlin Institute of Health, Berlin, Germany. ${ }^{4}$ Department of Anaesthesiology and Intensive Care Medicine, Philipps University Marburg, Marburg, Germany. ${ }^{5}$ Clinic of Anaesthesiology, Intensive Care Medicine and Pain Therapy, University Hospital Frankfurt, Frankfurt, Germany. ${ }^{6}$ Institute of Virology, Charité - Universitätsmedizin Berlin, corporate member of Free University Berlin, Humboldt-Universität zu Berlin, and Berlin Institute of Health and German Centre for Infection Research, Berlin, Germany. ${ }^{7}$ Division of Infectious Diseases, University Hospital and Medical Center Ulm, Ulm, Germany. ${ }^{8}$ Department of Anesthesiology and Critical Care Medicine, Carl Gustav Carus University Hospital, Technische Universität Dresden, Dresden, Germany. ${ }^{~ D e p a r t m e n t ~ o f ~ I n t e r n a l ~ M e d i c i n e ~ V ~-~ P n e u m o l o g y, ~ A l l e r g o l o g y, ~ I n t e n s i v e ~ C a r e ~ M e d i c i n e, ~ S a a r l a n d ~ U n i v e r s i t y ~ H o s p i t a l, ~ H o m b u r g, ~ G e r m a n y . ~}{ }^{10} \mathrm{Department}$ of Internal Medicine III, Hospital of Karlsruhe, Karlsruhe, Germany. "'Department of Gastroenterology, Hepatology, Pneumology and Infectious Diseases, Klinikum Stuttgart, Stuttgart, Germany. ${ }^{12 D e p a r t m e n t}$ of Anesthesiology and Critical Care, Medical Center - University of Freiburg, Faculty of Medicine, University of Freiburg, Freiburg, Germany. ${ }^{13}$ Medical Clinic I, Klinikum Landshut, Landshut, Germany. ${ }^{14}$ Department of Anesthesiology and Intensive Care Medicine, University Hospital Tübingen, Tübingen, Germany. ${ }^{15}$ Department of Anaesthesiology and Surgical Intensive Care Medicine, University of Heidelberg, University Medical Centre Mannheim, Mannheim, Germany. ${ }^{16}$ Institute of Immunology and Transfusion Medicine, University Hospital Greifswald, Greifswald, Germany. ${ }^{17}$ Institute of Epidemiology and Medical Biometry, Ulm University, Ulm, Germany. ${ }^{18}$ Institute of Transfusion Medicine and Immunohematology, German Red Cross Blood Transfusion Service Baden-Württemberg-Hessen, Frankfurt, Cermany. ${ }^{19} \mathrm{All}$ members of the CAPSID Clinical Trial Group are listed in Supplemental Acknowledgments (supplemental material available online with this article; https://doi.org/10.1172/JC1152264DS1).

BACKGROUND. COVID-19 convalescent plasma (CCP) has been considered a treatment option for COVID-19. This trial assessed the efficacy of a neutralizing antibody containing high-dose CCP in hospitalized adults with COVID-19 requiring respiratory support or intensive care treatment.

METHODS. Patients $(n=105)$ were randomized 1:1 to either receive standard treatment and 3 units of CCP or standard treatment alone. Control group patients with progress on day 14 could cross over to the CCP group. The primary outcome was a dichotomous composite outcome of survival and no longer fulfilling criteria for severe COVID-19 on day 21.

RESULTS. The primary outcome occurred in $43.4 \%$ of patients in the CCP group and $32.7 \%$ in the control group $(P=0.32)$. The median time to clinical improvement was 26 days in the CCP group and 66 days in the control group $(P=0.27)$. The median time to discharge from the hospital was 31 days in the CCP group and 51 days in the control group $(P=0.24)$. In the subgroup that received a higher cumulative amount of neutralizing antibodies, the primary outcome occurred in $56.0 \%$ of the patients (vs. $32.1 \%$ ), with significantly shorter intervals to clinical improvement ( 20 vs. 66 days, $P<0.05$ ) and to hospital discharge ( 21 vs. 51 days, $P=0.03$ ) and better survival (day-60 probability of survival $91.6 \%$ vs. $68.1 \%, P=0.02$ ) in comparison with the control group.

CONCLUSION. CCP added to standard treatment was not associated with a significant improvement in the primary and secondary outcomes. A predefined subgroup analysis showed a significant benefit of CCP among patients who received a larger amount of neutralizing antibodies.

TRIAL REGISTRATION. ClinicalTrials.gov NCT04433910.

FUNDING. Bundesministerium für Gesundheit (German Federal Ministry of Health): ZMVI1-2520C0R802.

Conflict of interest: Victor $M$. Corman is named together with Euroimmun on a patent application filed recently regarding the diagnosis of SARS-CoV-2 by antibody testing ("Methods and reagents for diagnosis of SARS-CoV-2 infection." Patent application no. EP3809137A1)

Copyright: (5) 2021, American Society for Clinical Investigation.

Submitted: June 14, 2021; Accepted: August 26, 2021; Published: October 15, 2021.

Reference information: J Clin Invest. 2021;131(20):e152264.

https://doi.org/10.1172/JCl152264.

\section{Introduction}

COVID-19 convalescent plasma (CCP) from patients who have recovered from a SARS-CoV-2 infection has become one of the treatment options for severe COVID-19 $(1,2)$. It has been broadly used in an expanded access program (3) in the United States, and preliminary reports on signals of efficacy and safety led to an Emergency Use Authorization in the United States in August 


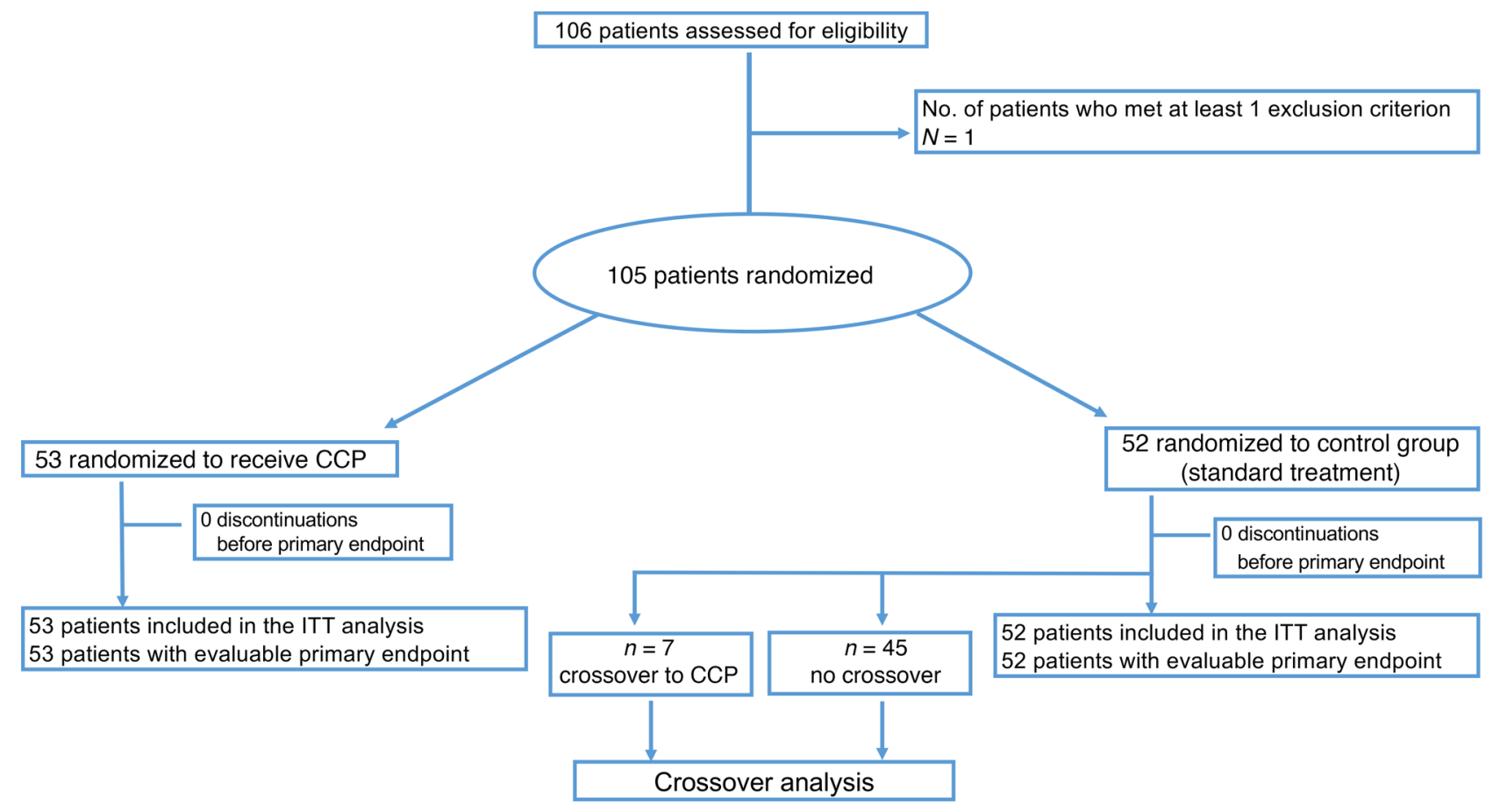

Figure 1. Patient enrollment and treatment assignment. ITT, intention to treat.

$2020(4,5)$. A large number of clinical trials on CCP have been initiated since the start of the pandemic (6-19). Clinical trial outcomes assessing efficacy have been mixed. A recent systematic review and meta-analysis concluded that CCP compared with placebo or standard of care was not significantly associated with a decrease in all-cause mortality or with any other benefit for other clinical outcomes $(18,19)$. However, several limitations were noted: risk of bias, insufficient reporting of clinical outcomes other than all-cause mortality, and limited data to perform subgroup analyses. The certainty of evidence was considered low to moderate for all-cause mortality and low for other outcomes (18). In contrast, another meta-analysis concluded that patients with COVID-19 transfused with CCP exhibited a lower mortality rate compared with patients receiving standard treatments, and it showed that early transfusion of higher-titer plasma is associated with lower patient mortality (20). The volume of CCP transfused was low in some of the trials $(6,7,9,10$, $12,14)$, and the content of antibodies in CCP units was poorly characterized or only measured post hoc in some of the trials. Therefore, it is important to gather data from further controlled clinical trials using well-defined CCP.

Here we present the results of a randomized, prospective, open-label, multicenter clinical trial of CCP compared with standard of care in hospitalized patients requiring supplemental oxygen or ventilation support or intensive care treatment (CAPSID trial). It includes some unique features: In addition to survival, a number of other outcomes are reported based on detailed definitions. Patients in the CCP group received 3 units of plasma over a period of 5 days, i.e., a scheduled volume of about $850 \mathrm{~mL}$ CCP, which is substantially higher than the CCP volume administered in other trials. Neutralizing SARS-CoV-2 antibodies were analyzed in detail both in the CCP products and in the recipients.

\section{Results}

Study population. A total of 106 patients were enrolled to this clinical trial, and 105 patients were randomized to either the CCP group $(n=53)$ or the control group $(n=52)$ (Figure 1$)$. All 105 patients were included in the intention-to-treat analysis.

Baseline demographics and clinical characteristics are shown in Tables 1 and 2. A minority of patients were female (26.7\%) and the majority were male (73.3\%). The median age was 60 years (IQR 53-66). A majority of patients $(89.5 \%)$ had a coexisting condition at entry into the trial (56.2\% hypertension, $31.4 \%$ diabetes, $21.9 \%$ cardiovascular disease). The median BMI was $29.4 \mathrm{~kg} / \mathrm{m}^{2}$. The percentage of patients receiving supplemental oxygen or noninvasive ventilation (score 4 and 5 on the WHO ordinal severity scale) or invasive ventilation (score 6 and 7 ) was $59.1 \%$ and $34.3 \%$, respectively. The time from symptom onset of the SARS-CoV-2 infection to randomization was 7 days (IQR 4-10). SARS-CoV-2 PCR from nasopharyngeal swabs was still positive in $94.3 \%$ of patients at baseline. Neutralizing SARS-CoV-2 antibodies were present in $78.9 \%$ of patients with available information at baseline (median titer among those with detectable antibodies 1:160, IQR 1:80 to 1:640; Table 2). The proportion of patients with detectable neutralizing SARS-CoV-2 antibodies was higher among those with longer interval ( $>7$ days) since onset of symptoms compared with those with shorter interval ( $\leq 7$ days) $(89.1 \%$ vs. $69.4 \%)$.

Patients were also classified by their inflammation markers at baseline (Table 2). Among the patients with high inflammation markers and low inflammation markers the proportion of patients 
Table 1. Baseline demographics and clinical characteristics

\begin{tabular}{|c|c|c|c|}
\hline & $\begin{array}{c}\text { CCP group } \\
(n=53)\end{array}$ & $\begin{array}{l}\text { Control group } \\
(n=52)\end{array}$ & $P$ value \\
\hline \multicolumn{4}{|l|}{ Demographic and clinical characteristics } \\
\hline Median age, years (IQR) & $59(53-65)$ & $62(55-66)$ & 0.24 \\
\hline Female & $11(20.8)$ & $17(32.7)$ & \\
\hline Male & $42(79.3)$ & $35(67.3)$ & \\
\hline $\mathrm{BMI}, \mathrm{kg} / \mathrm{m}^{2}$ (IQR) & $29.4(27.6-33.4)$ & $29.1(25.6-31.5)$ & 0.07 \\
\hline No other disease & $7(13.2)$ & $4(7.7)$ & \\
\hline $\mathrm{BMI}>30 \mathrm{~kg} / \mathrm{m}^{2}$ & $28(52.8)$ & $29(55.6)$ & \\
\hline Hypertension & $31(58.5)$ & $28(53.9)$ & \\
\hline Cardiovascular disease & $12(22.6)$ & $11(21.2)$ & \\
\hline Diabetes & $18(34.0)$ & $15(28.9)$ & \\
\hline COPD, asthma, other pulmonary disease & $8(15.1)$ & $9(17.3)$ & \\
\hline Point scale at study entry, $n(\%)$ & & & 0.68 \\
\hline 3 & $4(7.6)$ & $3(5.8)$ & \\
\hline 4 & $5(9.4)$ & $8(15.4)$ & \\
\hline 5 & $28(52.8)$ & $21(40.4)$ & \\
\hline 6 & $3(5.7)$ & $3(5.8)$ & \\
\hline 7 & $13(24.5)$ & $17(32.7)$ & \\
\hline Respiratory rate, breaths/min (IQR) & $24(20-30)$ & $23(18-30)$ & 0.82 \\
\hline Median time from symptom onset of the SARS-CoV-2 infection to randomization, days (IQR) & $7(2-9)$ & $7(5-10.5)$ & 0.07 \\
\hline Median time from hospitalization to randomization, days (IQR) & $1(1-3)$ & $2.5(1-5)$ & 0.02 \\
\hline \multicolumn{4}{|l|}{ Prior/concomitant medication, $n$ (\%) } \\
\hline Antiviral drug ${ }^{A}$ & $23(43.4)$ & $24(46.2)$ & \\
\hline PLT transfusion & $1(1.9)$ & $4(7.7)$ & \\
\hline FFP transfusion & $7(13.2)$ & $9(17.3)$ & \\
\hline No concomitant medication & $2(3.8)$ & $2(3.9)$ & \\
\hline \multicolumn{4}{|l|}{ SARS-CoV-2 status at baseline } \\
\hline SARS-CoV-2 PCR nasopharyngeal swab, $n(\%)$ & & & 0.62 \\
\hline Positive & $49(92.5)$ & $50(96.2)$ & \\
\hline Negative & $3(5.7)$ & $1(1.9)$ & \\
\hline Missing & $1(1.9)$ & $1(1.9)$ & \\
\hline
\end{tabular}

Ancluding remdesivir in 20 patients (37.7\%) in the CCP group and 22 patients $(42.3 \%)$ in the control group. COPD, chronic obstructive pulmonary disease; FFP, fresh frozen plasma (nonimmune plasma, not for indication of passive immunotherapy against SARS-CoV-2); PLT, platelet concentrate; RBC, red blood cell concentrate.

with less severe disease (score 3 and 4 on the WHO ordinal severity scale) was $9.8 \%$ and $29.2 \%$, respectively.

Overall, the CCP group and the control group were similar in terms of demographic characteristics and disease severity as assessed by the distribution on the ordinal severity scale, the type of ventilation support, and the laboratory results at baseline, except ferritin levels at baseline and the interval from hospitalization to randomization (Tables 1 and 2).
Study treatment. Fifty-two of 53 patients (98.1\%) randomized to CCP received all 3 planned CCP transfusions with a median total volume of $846 \mathrm{~mL}$ (IQR 824$855 \mathrm{~mL}$ ). The median neutralization titer in the plaque reduction neutralization test (50\% inhibition) (PRNT50) of CCP was 1:160 (IQR 1:80 to 1:320; Supplemental Figure 1 ; supplemental material available online with this article; https://doi.org/10.1172/JCI152264DS1). Themediantransfused 
Table 2. Humoral immune response and laboratory values at baseline

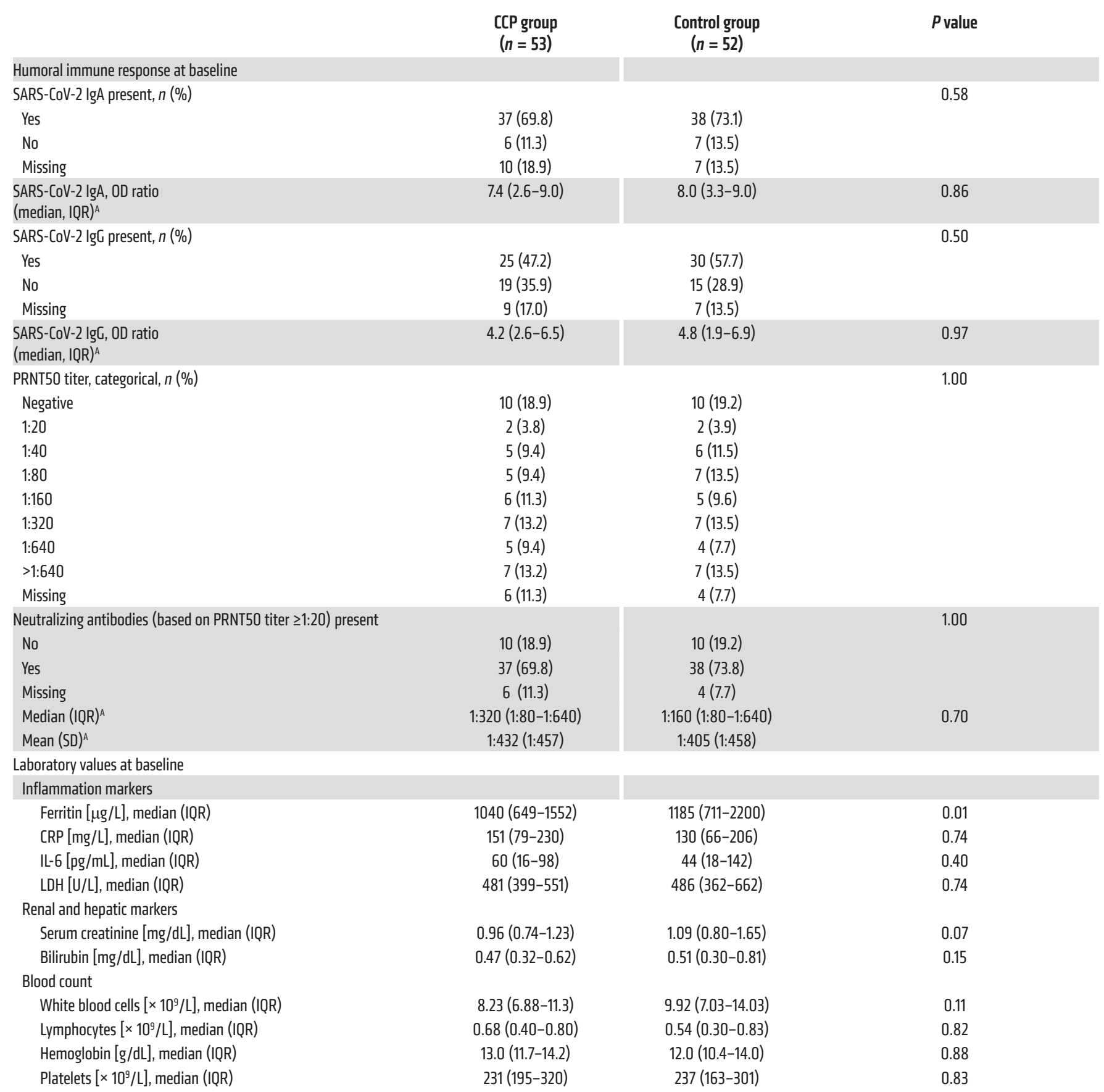

AThis descriptive analysis included only patients with detectable antibodies (PRNT50 21:20). CRP, C-reactive protein; LDH, lactate dehydrogenase; PRNT50, neutralization titer in the plaque reduction neutralization test ( $50 \%$ inhibition).

neutralizing units per patient were 6768 (IQR 3424-13,520). The majority of patients (96.2\%) received only ABO-identical transfusions, 1 patient each with type $\mathrm{A}$ and type $\mathrm{B}$ received $\mathrm{AB}$ plasma, and 50 patients of $53(94.3 \%)$ received all 3 plasma units from 1 donor collected in either 1 or 2 plasmapheresis sessions (79.3\% and $15.1 \%$, respectively). Crossover patients received a median total volume of $837 \mathrm{~mL}$ (IQR 738-872 mL), and all but 1 crossover patients received all 3 CCP transfusions.
Primary clinical outcome. There was no significant difference in the dichotomous composite primary outcome at day 21 (no longer requiring ventilation support or ICU treatment and no tachypnea, i.e., respiratory rate $<30$ per minute): $43.4 \%$ of patients in the CCP group and $32.7 \%$ in the control group reached the primary outcome $(P=0.32$; Table 3$)$. Among those who received a low or high cumulative amount of neutralizing units, the primary outcome occurred in $32.1 \%$ and $56.0 \%$, respectively (Table 4 ). 
Table 3. Primary outcome at day 21

\begin{tabular}{|c|c|c|c|}
\hline & $\begin{array}{l}\text { CCP group } \\
(n=53)\end{array}$ & $\begin{array}{c}\text { Control group } \\
(n=52)\end{array}$ & $P$ value \\
\hline & & & 0.32 \\
\hline Treatment success, $n(\%)$ & $23(43.4)$ & $17(32.7)$ & \\
\hline Treatment failure, $n(\%)$ & $30(56.6)$ & $35(67.3)$ & \\
\hline
\end{tabular}

Table 4. Primary outcome at day 21 by transfused neutralizing units ${ }^{A}$

\begin{tabular}{|c|c|c|c|}
\hline & \multicolumn{2}{|c|}{$\begin{array}{l}\text { CCP group } \\
(n=53)\end{array}$} & \multirow[t]{2}{*}{$\begin{array}{l}\text { Control group } \\
\quad(n=52)\end{array}$} \\
\hline & $\begin{array}{l}\text { Low neutralizing units }{ }^{A} \\
\qquad(n=28)\end{array}$ & $\begin{array}{l}\text { High neutralizing units }{ }^{A} \\
\qquad(n=25)\end{array}$ & \\
\hline Treatment success, $n(\%)$ & $9(32.1)$ & $14(56.0)$ & $17(32.7)$ \\
\hline Treatment failure, $n(\%)$ & $19(67.9)$ & $11(44.0)$ & $35(67.3)$ \\
\hline
\end{tabular}

${ }^{A}$ See Methods for definition of neutralizing units. CCP group was divided by the cumulative amount of neutralizing units per patient (all 3 CCP transfusions) into a low-neutralizing unit group ( $\leq$ median) and a high-neutralizing unit group (> median).
The majority of patients (81.0\%) experienced at least one AE. Neither the frequency of AEs nor the worst AE grade significantly differed between the groups $(P=0.62$ and $P=0.18$, respectively.; Supplemental Table 1). The outcome of AEs was similar between the groups. The proportion of patients with serious AEs (SAEs) was $41.5 \%$ in the CCP group and $48.1 \%$ in the control group. Number of SAEs and reasons for classification as SAE are summarized in Supplemental Table 1.

The case fatality rate at day 21 , day 35 , and day 60 was $14.3 \%, 21.0 \%$, and $26.7 \%$, respectively, without a significant difference between the groups (Supplemental Table 2). The probability of overall survival at day 60 was $77.9 \%$ (95\% CI 63.6\%-87.1\%) in the CCP group and $68.1 \%$ (95\% CI $53.3 \%-79.1 \%)$ in the control group $(P=0.21$, log-rank test; Figure 2B). The median time to hospital discharge was 31 days (IQR 16-NR) in the CCP group and 51 days (IQR 20-NR) in Among those with low and high inflammation markers at entry ( $n=48$ and $n=51$, respectively), the primary outcome occurred in 26 of $48(54.2 \%)$ and 13 of 51 (25.5\%) patients, respectively ( $P=$ 0.004, Fisher's exact test, explorative analysis; Table 5). Among the patients without or with neutralizing antibodies at baseline ( $n=20$ and $n=75$, respectively), the primary outcome occurred in 5 of $20(25.0 \%)$ and 32 of $75(42.7 \%)$ patients, respectively $(P$ $=0.20$, Fisher's exact test, explorative analysis; Table 6). Among those with or without invasive ventilation or extracorporeal membrane oxygenation (ECMO) at entry ( $n=36$ and $n=69$, respectively), the primary outcome occurred in 5 of $36(13.9 \%)$ and 35 of 69 (50.7\%) patients, respectively $(P<0.001$, Fisher's exact test, explorative analysis; Table 7). However, within these subgroups (Tables 5-7) the outcome did not significantly differ between the CCP group and the control group.

Secondary clinical outcome. The median time to clinical improvement by $\geq 2$ points on the ordinal severity scale was 26 days (IQR 15-NR [not reached]) in the CCP group and 66 days (IQR 13-NR) in the control group $(P=0.27$, log-rank test; Figure 2A). the control group $(P=0.24, \log$-rank test; Figure $2 \mathrm{C})$.

The median time to discharge from ICU was 29 days (IQR 9-NR) in the CCP group and 42 days (IQR 12-NR) in the control group $(P=0.39$, log-rank test; Figure 2D).

The distribution of clinical outcomes according to the ordinal severity scale is shown in Figure 3. On day 35, 65.3\% of patients with available follow-up information in the CCP group and $44.0 \%$ in the control group were discharged from hospital or no longer required supplemental oxygen $(P=0.04$, Fisher's exact test, exploratory analysis). The proportion of patients still requiring supplemental oxygen or noninvasive or invasive ventilation on day 35 was $18.4 \%$ in the CCP group and $28.0 \%$ in the control group.

The median time to first negative SARS-CoV-2 PCR from nasopharyngeal swab was 7 days (IQR 4-17 days) in the CCP group and 8 days (IQR $5-21$ days) in the control group $(P=0.38$, log-rank test; Supplemental Figure 2A).

The secondary time to event outcomes by transfused neutralizing units is summarized in Supplemental Table 3. Among those patients in the CCP group who received a high cumulative amount of neutralizing units compared with those with a low amount, the

\section{Table 5. Primary outcome at day 21 by inflammation markers ${ }^{A}$}

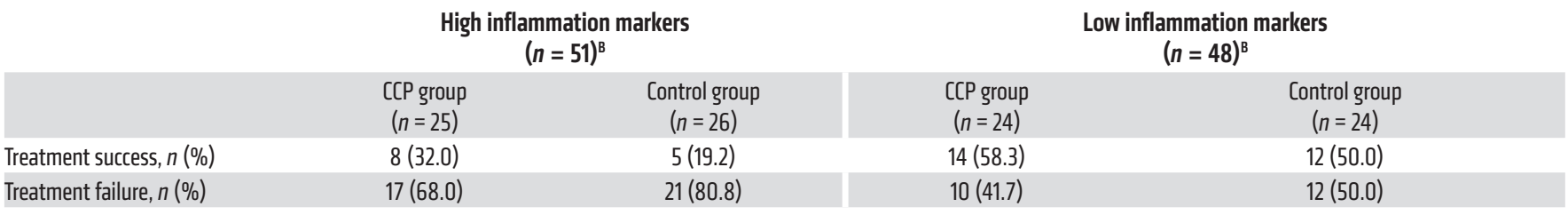

ASee Methods section for definition of high and low inflammation markers. The patient group was divided into a low-inflammation marker group and a high-inflammation marker unit group. In each of these groups the primary outcome for patients in the CCP group and the control group was compared. ${ }^{\mathrm{B}} \mathrm{Six}$ patients with either missing data on inflammation markers $(n=1)$ or intermediate inflammation markers $(n=5)$ are not included in this table. 
Table 6. Primary outcome at day 21 by presence/absence of SARS-CoV-2 neutralizing antibodies at baseline ${ }^{A}$

\begin{tabular}{|c|c|c|c|c|}
\hline & \multicolumn{2}{|c|}{$\begin{array}{l}\text { Anti-SARS-CoV-2 neutralizing antibodies at baseline: positive } \\
\qquad(n=75)^{\mathrm{B}}\end{array}$} & \multicolumn{2}{|c|}{$\begin{array}{l}\text { Anti-SARS-CoV-2 neutralizing antibodies at baseline: negative } \\
\qquad(n=20)^{\mathrm{B}}\end{array}$} \\
\hline & $\begin{array}{l}\text { CCP group } \\
(n=37)\end{array}$ & $\begin{array}{l}\text { Control group } \\
(n=38)\end{array}$ & $\begin{array}{l}\text { CCP group } \\
(n=10)\end{array}$ & $\begin{array}{l}\text { Control group } \\
(n=10)\end{array}$ \\
\hline Treatment failure, $n(\%)$ & $20(54.0)$ & $23(60.5)$ & $6(60.0)$ & $9(90.0)$ \\
\hline
\end{tabular}

${ }^{A}$ Presence of anti-SARS-CoV-2 neutralizing antibodies with titers of PRNT50 $\geq 1: 20$ at baseline. ${ }^{\mathrm{B}}$ Ten patients with missing data on neutralizing antibodies (PRNT50) at baseline are not included in this table.

\section{Table 7. Primary outcome at day 21 by ventilation status at baseline}

\begin{tabular}{lcccc} 
& \multicolumn{2}{c}{$\begin{array}{c}\text { Patients without invasive ventilation/ECMO } \\
(\boldsymbol{n}=69)\end{array}$} & & \multicolumn{2}{c}{$\begin{array}{c}\text { Patients with invasive ventilation/ECMO } \\
(\boldsymbol{n}=36)\end{array}$} \\
& $\begin{array}{c}\text { CCP group } \\
(n=37)\end{array}$ & $\begin{array}{c}\text { Control group } \\
(n=32)\end{array}$ & $\begin{array}{c}\text { CCP group } \\
(n=16)\end{array}$ & $\begin{array}{c}\text { Control group } \\
(n=20)\end{array}$ \\
\hline Treatment success, $n(\%)$ & $21(56.8)$ & $14(43.8)$ & $2(12.5)$ & $3(15.0)$ \\
Treatment failure, $n(\%)$ & $16(43.2)$ & $18(56.3)$ & $14(87.5)$ & $17(85.0)$ \\
\hline
\end{tabular}

median time to clinical improvement was 20 days (IQR 11-NR) and 36 days (IQR 17-NR), respectively (Figure 4A and Supplemental Table 3). The median time to hospital discharge was 21 days (IQR 13-43) and 39 days (IQR 21-NR) (Figure 4C and Supplemental Table 3), the median time to discharge from ICU was 14 days (IQR 7-39) and 39 days (IQR 20-NR) (Figure 4D and Supplemental Table 3), and the median time to negative SARS-CoV-2 PCR was 5 days (IQR 3-15) and 14 days (IQR 5-19) (Supplemental Figure 2B and Table 3). Overall survival was higher among patients transfused with high amounts of neutralizing antibodies compared with the control group ( $P=0.02$; Figure $4 \mathrm{~B})$.

The secondary time to event outcomes by inflammation markers at baseline is summarized in Supplemental Table 4 and Supplemental Figure 3. For those patients with high inflammation markers the median time to clinical improvement was not reached (Supplemental Table 4 and Supplemental Figure 3A). Among patients with low inflammation markers at baseline, the median time to clinical improvement was 19 days (IQR 8-NR) in the CCP group and 41 days (IQR 13-NR) in the control group. Among patients with high inflammation markers, the time to discharge from hospital was 41 days (IQR 21-NR) in the CCP group and was not reached in the control group (Supplemental Table 4 and Supplemental Figure 3B). Among patients with low inflammation markers, the median time to hospital discharge was 24 days (IQR 11-NR) in the CCP group and 26 days (IQR 12-NR) in the control group.

The secondary time to event outcomes by presence of neutralizing antibodies at baseline and by the ventilation status at baseline is summarized in Supplemental Tables 5 and 6.

Crossover patients. Baseline characteristics of crossover patients are summarized in Supplemental Table 7. None of the crossover patients achieved clinical improvement and hospital discharge, and all 7 patients died (Supplemental Figure 4). We compared the crossover patients with a propensity scorematched subset of patients from the initial CCP group. Patients matched according to ventilation status on day +14 and baseline characteristics (Supplemental Table 8) showed no difference in overall survival (Supplemental Figure 5B). In contrast, a subset matched for baseline characteristics only (Supplemental Table 9) showed a significant difference in overall survival (Supplemental Figure 5A).

\section{Discussion}

This trial found no statistically significant difference in the primary outcome, a dichotomous composite outcome of survival without ventilation support at day 21 after treatment with CCP compared with standard treatment in hospitalized patients who required supplemental oxygen, noninvasive or invasive ventilation, or ICU treatment. Use of CCP was also not associated with significant improvement in the secondary outcomes time to clinical improvement, time to ICU discharge, time to hospital discharge, and case fatality rate.

In 2 subgroup analyses, we were able to demonstrate that the primary outcome was better in patients with low inflammation markers at baseline and in patients not requiring invasive ventilation or ECMO at baseline. However, in the subgroup analysis the primary and secondary outcomes did not differ significantly between the treatment groups. A better outcome of patients not requiring invasive ventilation is in line with other reports $(3,6)$.

So far there is no consensus on a minimum antibody titer in CCP - mainly owing to a lack of standardization of assays for SARS-CoV-2 neutralizing antibodies. While there is an increasing number of studies comparing results of total binding antibodies $(21,22)$, the comparability of assays for neutralizing antibodies is rather limited. Therefore, it is difficult to compare the neutralizing capacity of CCP products used in the different clinical trials. In addition to direct antibody-mediated virus neutralization, other mechanisms of action based on Fc-dependent antibody functions $(23,24)$ as well as cellular immunological effects $(25)$ may have implications for therapeutic efficacy of CCP. Longitu- 
A

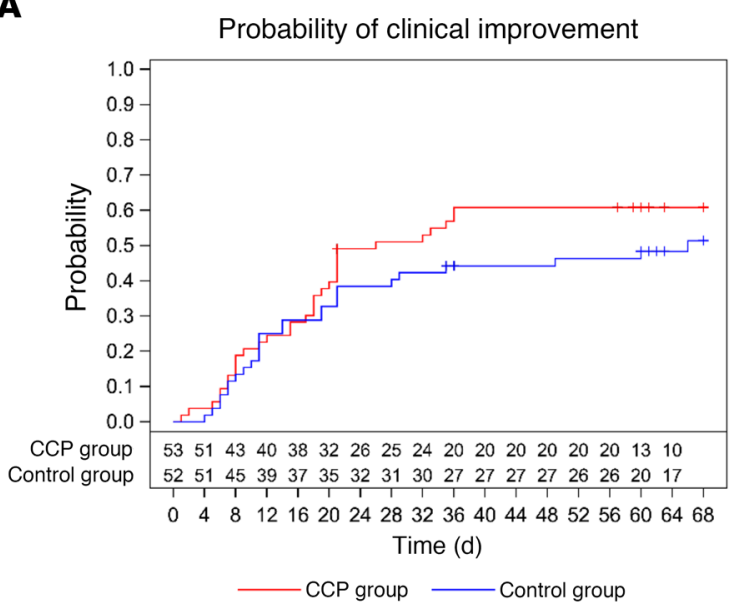

C

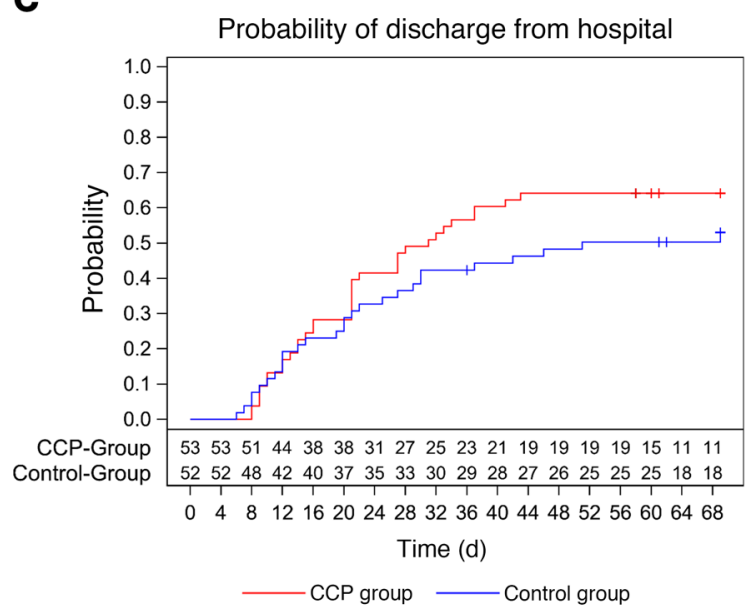

B

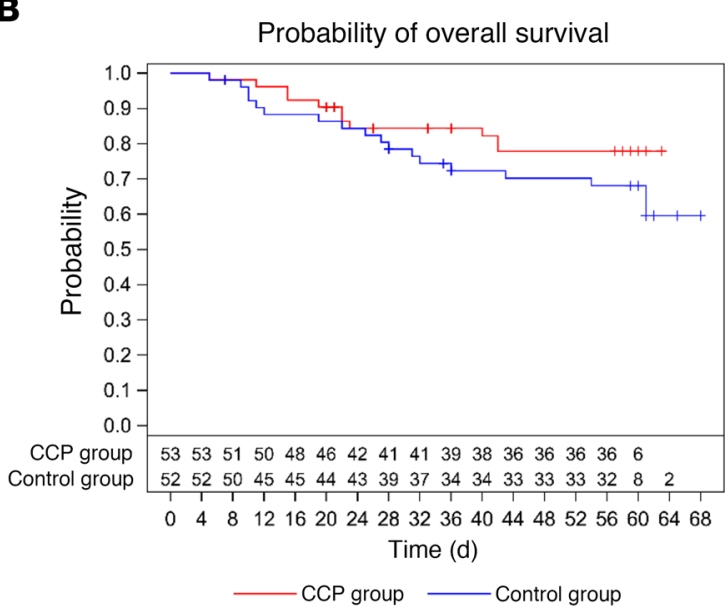

D

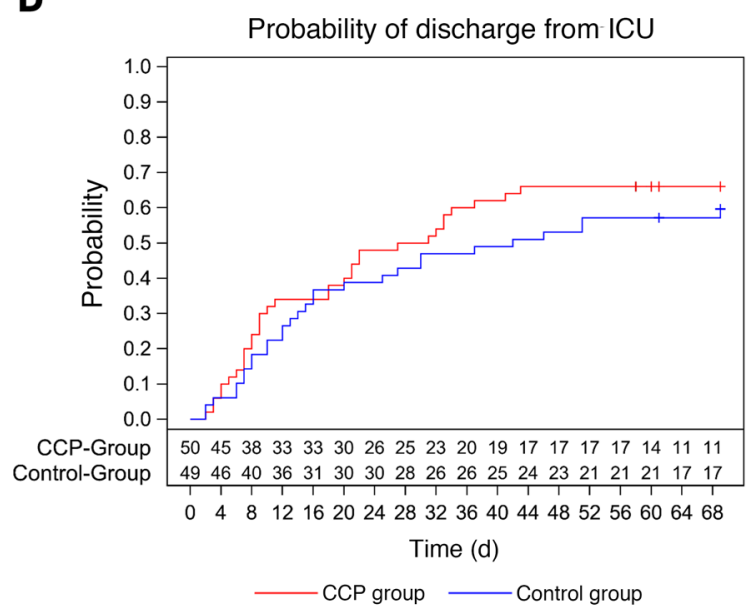

Figure 2. Occurrence of secondary outcomes. Kaplan-Meier cumulative estimates of probability are shown. (A) The key secondary outcome time to clinical improvement compared in the CCP group (red line) and control group (blue line). Censored patients are indicated by "+". $P=0.27$ (log-rank test). (B) Probability of overall survival compared in the CCP group (red line) and control group (blue line). Censored patients are indicated by " $+" . P=0.21$ (log-rank test). (C) Probability of discharge from hospital compared in the CCP group (red line) and control group (blue line). Censored patients are indicated by "+". $P=0.24$ (log-rank test). (D) Probability of discharge from ICU compared in the CCP group (red line) and control group (blue line). Censored patients are indicated by “+". $P=0.39$ (log-rank test).

dinal analysis of humoral immunity against SARS-CoV-2 spike in convalescent individuals demonstrated a decrease of IgM and neutralization capacity while IgG levels and Fc effector functions remained relatively stable (26). Also, the total volume of transfused CCP matters. In this trial the median total dose of plasma per patient was $846 \mathrm{~mL}$, which is higher than in other published clinical trials that administered a total CCP volume of $200 \mathrm{~mL}$ (6), 200-250 mL (17), $250 \mathrm{~mL}$ (14), $300 \mathrm{~mL}$ (9), 400 (7, 10, 12, 15), $500 \mathrm{~mL}(3,11,13)$, and $550 \mathrm{~mL}(16)$. Despite a high CCP volume the CAPSID trial failed to demonstrate a significant effect on the primary outcome. However, there was a signal of benefit from CCP in a prespecified subgroup analysis of patients who were treated with a high amount of neutralizing antibodies. The primary outcome was numerically better and the time to clinical improvement, time to ICU discharge and hospital discharge, and overall survival were significantly better (exploratory analysis) in the subgroup that received high amounts of neutralizing units compared with the control group. Previous studies have noted benefit from CCP therapy in early disease or the first 3 days of hospital admission $(3,14,27)$. Thus, in this context the first CCP transfusion in the row of 3 transfusions over 5 days might be the most consequential. Therefore, we performed an analysis that included only the amount of neutralizing antibodies of the first CCP transfusion (data not shown). This analysis also demonstrated a significantly shorter time to clinical improvement and shorter time to hospital discharge and significantly better overall survival among patients who received high amounts of neutralizing units with the first CCP transfusion.

For the findings in this trial, 3 characteristics of the patient population might be of relevance: (a) the population included patients with respiratory distress in a broad range from supplemental oxygen to invasive ventilation (the latter subgroup comprising about $34 \%$ ); (b) the median interval from onset of symptoms to randomization was 7 days; and (c) the majority of patients already had neutralizing antibodies at baseline. Other trials that also included hospitalized patients requiring respiratory support at least in a 


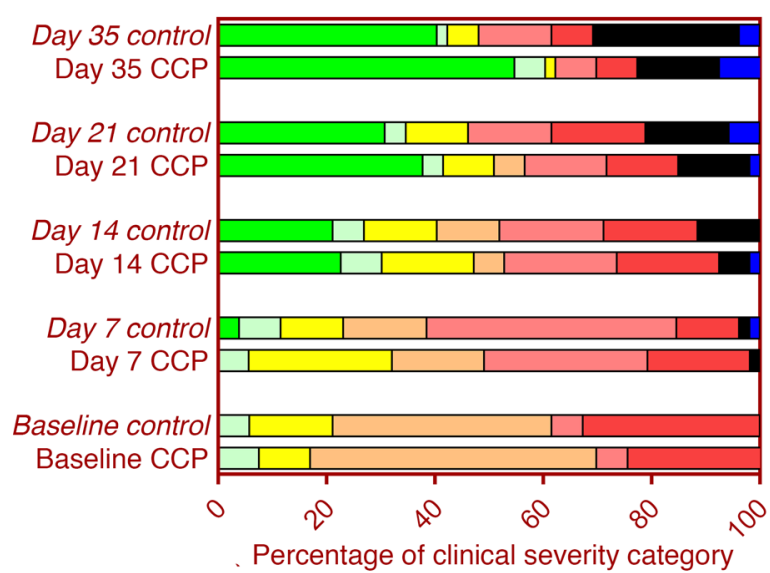

Ordinal severity scale

Missing

8 - Death

$\square$ - invasive ventilation* and orgain failure

6 - Invasive ventilation*

5 - Noninvasive ventilation*

4 - Supplementary oxygen*

3 - No supplementary oxygen*

$\leq 2$ (discharge)
Figure 3. Clinical outcomes according to ordinal severity scale. The distribution of the clinical status according to the ordinal severity scale at baseline, day 7 , day 14 , day 21 , and day 35 is shown for the CCP group and control group according to initial randomization, i.e., the 7 patients with crossover to receive CCP on days 15,17 , and 19 remain in the control group. ${ }^{*}$ Hospitalized. proportion of patients also did not report significant differences in clinical improvement or all-cause mortality $(6-13,16)$. However, in the CAPSID trial, the proportion of patients with life-threatening disease, i.e., requiring invasive ventilation or ECMO, was higher than in most other trials $(6-13,16)$. This trial included a larger proportion of patients with poor prognosis - based on proportion of patients with invasive ventilation, high inflammation markers, and comorbidities that are associated with poor outcome (28).

Despite a short median interval of 7 days between onset of symptoms and randomization, a majority of patients had already mounted an immune response, and the median PRNT50 titer was 1:160 - even before the transfusion of the first CCP unit. Similar findings were reported from other trials $(8,9,13,16)$, and one trial has even been stopped early because of this observation (9). An observational study reported an association between absent or low levels of antibodies against the spike protein of SARS-CoV-2 at ICU admission and an increased risk of mortality, higher frequency of antigenemia, and higher viral RNA loads in plasma (29). It was suggested that profiling anti-SARS-CoV-2 spike antibodies at ICU admission could help to better identify those patients who would potentially benefit from CCP (29).

Thus, late administration of CCP to patients who already had progressed to severe COVID-19 requiring ventilation support or ICU treatment and who already had developed neutralizing antibodies did not significantly improve all-cause mortality or time to clinical improvement with an effect size that was the basis for the sample size calculation of this trial. In contrast, early treatment with high-titer CCP within 72 hours of onset of mild COVID-19 symptoms reduced the risk of progression to severe respiratory disease by $48 \%$ (14). This is in line with our observations in the subgroup analysis by the cumulative amount of transfused neutralizing antibodies and other reports $(3,17,27)$.

There was no signal that frequency, severity, type, and outcome of AEs or SAEs in the CCP group differed from those in the control group. Rather, there was a tendency toward a lower number of AEs and lower number of SAEs in the CCP group. Thus, like other studies $(6-13,17,30)$, this trial does not raise concerns that there are new safety issues if plasma is given in the proinflammatory and prothrombotic state of severe COVID-19.

A specific feature of this trial that differs from other trials is the option of crossover of patients from the control group to receive
CCP if they presented with progressive COVID-19 on day 14 . This should address the question of whether even late administration of CCP in progressive COVID-19 can improve outcome. Only 7 patients were switched, none had achieved clinical improvement, and all died. The baseline characteristics of the patients who were switched to the CCP arm did not differ from those of the control group patients who were not switched to receive CCP. The crossover patients were in a poor clinical condition on day $14-$ just based on the fact that they were eligible for crossover, which indicated progressive respiratory disease. The comparison of crossover patients with propensity score-matched subgroups from the initial CCP group suggests that the very poor outcome of this small subgroup represents a selection of patients with poor prognosis and unfavorable clinical course irrespective of treatment. Our observation does not support the use of CCP as a last resort in progressive patients.

Potential limitations of this trial include the following: (a) The design was open-label. However, the primary outcome was based on objective criteria and was hardly influenced by the knowledge of the randomization group. (b) Because of the eligibility criteria and the primary outcome of this study, the results do not allow conclusions for patients not requiring hospitalization. (c) There were no stringent rules for the standard treatment, which was allowed in both groups. Standard treatment regarding ventilation procedures, corticosteroids, anticoagulation, and antiviral substances developed rapidly during the study period. A large proportion of patients in both groups had received corticosteroids, which might counteract CCP efficacy by inhibiting antibodydependent cytotoxicity or antibody-dependent phagocytosis as has been shown in animal experiments (24). However, since a large proportion of patients (Table 1 ) had received corticosteroids, we cannot provide data from this trial regarding whether a temporal relationship between CCP transfusion and corticosteroids leads to a clinically relevant antagonism. There is no obvious difference in concomitant treatment with potential impact on the outcome of COVID-19. However, it is difficult to assess the non-pharmacological interventions, e.g., ventilation support (31), which are also relevant to the outcome of COVID-19. (d) The protocol did not prospectively define limits for the allowed interval between onset of symptoms and CCP transfusion (except a maximum time of 72 hours after start of mechanical ventilation). The median time 
A

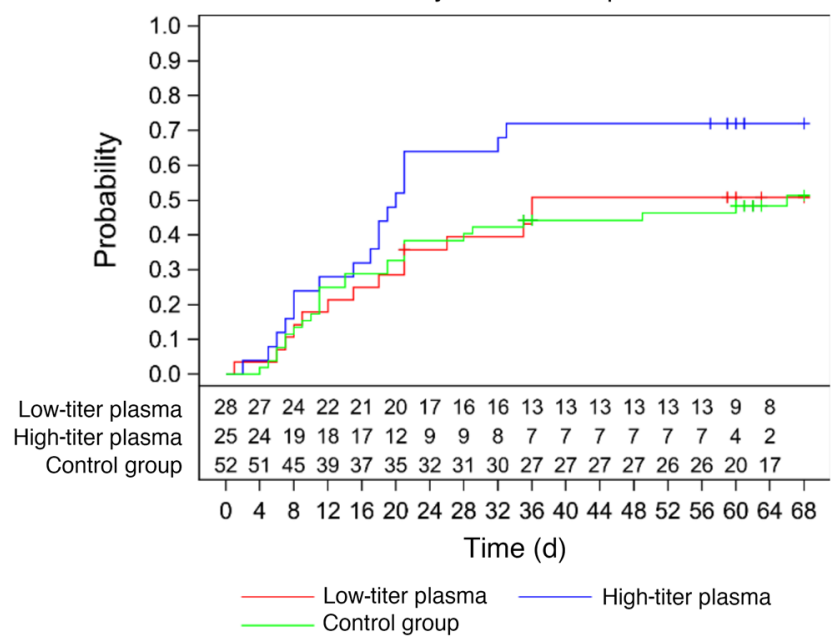

C

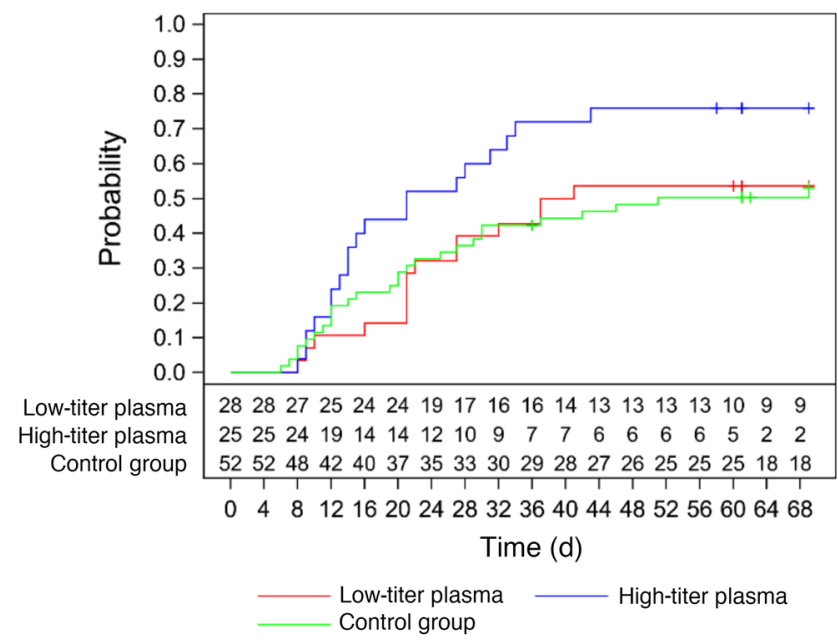

B

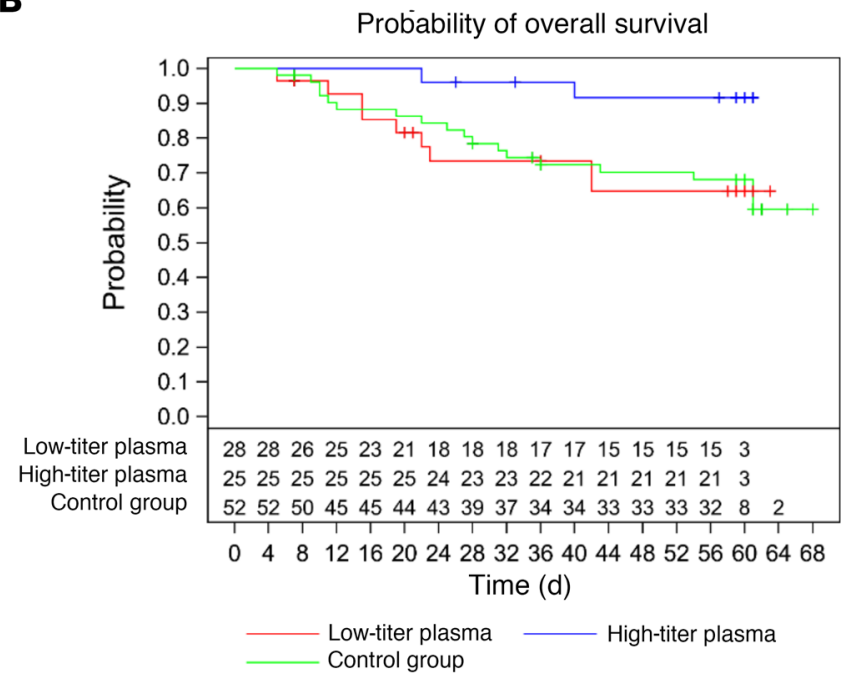

D

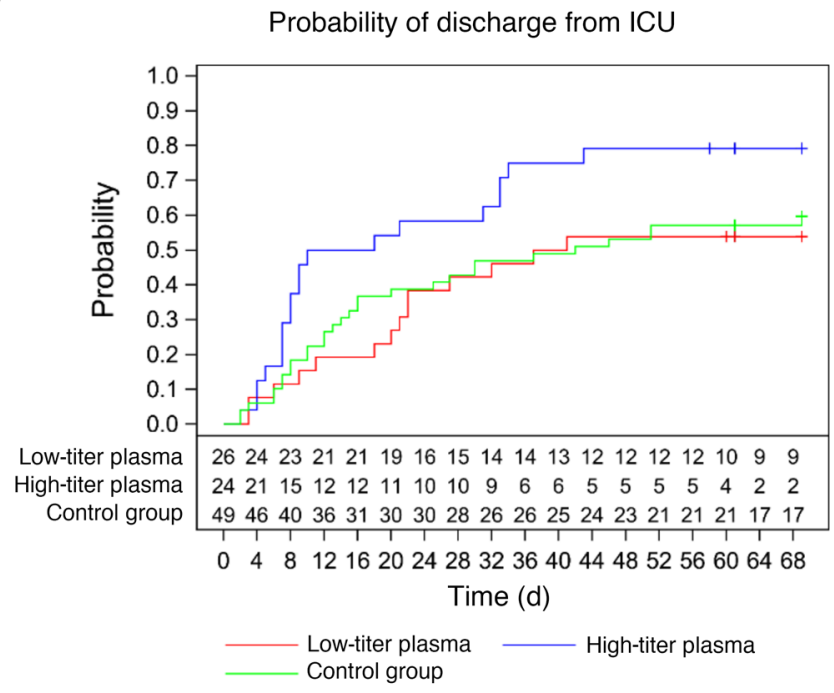

Figure 4. Occurrence of secondary outcomes by cumulative amount of transfused neutralizing units. Kaplan-Meier cumulative estimates of probability are shown. (A) The key secondary outcome time to clinical improvement compared in the CCP subgroup that received a low cumulative amount of neutralizing units (red line), the CCP subgroup that received a high cumulative amount of neutralizing units (blue line), and the control group (green line). Censored patients are indicated by "+". $P=0.0496$ (log-rank test, high amount vs. control group). (B) Probability of overall survival compared in the CCP subgroup that received a low cumulative amount of neutralizing units (red line), the CCP subgroup that received a high cumulative amount of neutralizing units (blue line), and the control group (green line). Censored patients are indicated by "+". $P=0.02$ (log-rank test, high amount vs. control group). (C) Probability of discharge from hospital compared in the CCP subgroup that received a low cumulative amount of neutralizing units (red line), the CCP subgroup that received a high cumulative amount of neutralizing units (blue line), and the control group (green line). Censored patients are indicated by " + ". $P=0.03$ (log-rank test, high amount vs. control group). (D) Probability of discharge from ICU compared in the CCP subgroup that received a low cumulative amount of neutralizing units (red line), the CCP subgroup that received a high cumulative amount of neutralizing units (blue line), and the control group (green line). Censored patients are indicated by “+". $P=0.04$ (log-rank test, high amount vs. control group).

between symptom onset and first CCP transfusion was 8 days (IQR 3.0-10.0 days). A subgroup analysis by the time between onset of symptoms and first transfusion did not demonstrate a significant difference in the primary outcome and the secondary efficacy outcomes. This does not contradict the conclusion from other trials $(3,14,27)$, since this trial was not powered to detect an impact of CCP timing on outcome. (e) The sample size calculation was based on the assumption that the primary outcome would be met by $40 \%$ of patients in the control group and $70 \%$ in the CCP group. In retrospect, this was an overestimated effect size and the study might have been underpowered. This limitation also applies to sample sizes of the subgroup analyses. The outcomes time to clinical improvement, time to hospital discharge, and overall survival show a consistent signal of better outcome in the CCP group. An improvement less than assumed in the sample size calculation for this clinical trial could still be clinically meaningful but would require a larger sample size.

In conclusion, among hospitalized adult patients with severe COVID-19, CCP added to standard therapy compared with standard therapy alone did not result in a statistically significant 
improvement of the primary outcome, i.e., survival free of ventilation support on day 21 and the key secondary outcome time to clinical improvement. Frequency and severity of adverse events did not differ between treatment groups. The consistent trend for a benefit across all primary and secondary outcomes among patients who received a higher amount of neutralizing antibodies provides a signal that better outcomes can be achieved by highdose CCP treatment combining very high titers of neutralizing antibodies with high CCP volumes. This should be addressed in further studies that focus on highly selected CCP with very high SARS-CoV-2 antibody titers.

\section{Methods}

Design. This was a multicenter, open-label randomized clinical trial to evaluate the efficacy and safety of CCP added to standard therapy (CCP group) versus standard therapy alone (control group) in hospitalized patients with COVID-19 (Figure 1). Patients in the control group with progressive COVID-19 on day 14 were eligible to switch to treatment with CCP (crossover group).

Patients. A total of 106 patients were recruited from 13 hospitals in Germany in the period from August 30, 2020, to December 24, 2020. Follow-up was completed on February 23, 2021.

Inclusion criteria were (a) SARS-CoV-2 infection confirmed by PCR (bronchoalveolar lavage, sputum, nasal and/or pharyngeal swab); (b) age $\geq 18$ years and $\leq 75$ years; (c) severe disease defined by at least 1 of the following: (i) respiratory rate $\geq 30$ breaths/min under ambient air, (ii) requirement of any type of respiratory support (defined as supplemental oxygen, noninvasive or invasive ventilation, or ECMO), (iii) need of treatment on ICU; and (d) written informed consent by patient or representative.

Exclusion criteria were (a) accompanying diseases other than COVID-19 with an expected survival time of less than 12 months; (b) previous treatment with any SARS-CoV-2 convalescent plasma; (c) opinion of the clinical team that progression to death is imminent and inevitable within the next 48 hours, irrespective of the provision of treatment; (d) interval $>72$ hours since start of mechanical ventilation; (e) not considered eligible for extracorporeal oxygenation support; (f) chronic obstructive pulmonary disease, stage 4; (g) lung fibrosis with usual interstitial pneumonia pattern in CT and severe emphysema; (h) chronic heart failure NYHA $\geq 3$ and/or preexisting reduction of left ventricular ejection fraction to $\leq 30 \%$; (i) shock of any type requiring $\geq 0.5 \mu \mathrm{g} / \mathrm{kg} / \mathrm{min}$ noradrenaline (or equivalent) or requiring more than 2 types of vasopressor medication for more than 8 hours; (j) liver cirrhosis Child C; (k) liver failure: bilirubin greater than $5 \times$ upper limit of normal (ULN) and elevation of alanine transaminase/aspartate transaminase (at least one greater than $10 \times$ $\mathrm{ULN}$ ); (l) any history of adverse reactions to plasma proteins; (m) known deficiency of IgA; (n) pregnancy; (o) breastfeeding (women); (p) volume overload until sufficiently treated; and (q) participation in another clinical trial with an investigational medicinal product.

None of the patients had received SARS-CoV-2 vaccination. None of the patients had a hematological malignancy, had received an organ transplant, or was on immunosuppressive agents (e.g., CD20 antibodies) other than corticosteroids (see Table 1 , concomitant medication) or azathioprine $(n=1)$.

Randomization. Patients $(n=105)$ were randomized using a Web-based system with a stratified 1:1 allocation ratio between each stratum (Figure 1). Patients were stratified prior to permutated block randomization by presence or absence of ventilation support, ECMO, or ICU treatment.

Sample size calculation. Sample size calculation was based on an $\alpha$ error of 0.05 , a power of 0.8 , a 2 -sided comparison, and an expected improvement of the primary outcome from $40 \%$ (control) to $70 \%$ (CCP). This resulted in a patient number of 48 patients per arm calculated by means of a Fisher's exact test. Assuming a dropout rate of $10 \%$, the overall number per arm was 53 patients. Sample size calculation was performed with $\mathrm{G}^{*}$ Power version 3.1.9.4 (https://www. apponic.com/developer/heinrich-heine-university-44943/).

Crossover from control group to CCP treatment. Clinical condition in all patients was evaluated on day 14. In case of progression on day 14 compared with baseline, patients in the control group could receive CCP. A patient switching to CCP on day 14 was considered as having failure of the primary outcome. Detailed criteria of progress for the crossover decision are described in Supplemental Methods.

SARS-CoV-2 antibody assays. A plaque reduction neutralization test (PRNT) and an ELISA for the detection of IgG and IgA against the spike protein of SARS-CoV-2 were performed as previously described $(21,32,33)$. Details are described in Supplemental Methods.

Convalescent plasma transfusions and standard treatment. Patients who had recovered from SARS-CoV-2 infection were recruited as donors. CCP was collected by plasmapheresis with a median collection volume of $850 \mathrm{~mL}$. The target volume of the CCP units was $250-325 \mathrm{~mL}$. The majority of donors had a mild or moderate course of COVID-19. Titers of neutralizing antibodies were measured by a PRNT, and CCP contained a PRNT50 titer of at least 1:20. A series of 144 donors (41\% female, $59 \%$ male; median age 40 years) who were not vaccinated against SARSCoV-2 underwent 319 plasmapheresis procedures, providing a median collection volume of $850 \mathrm{~mL}$ and a mean number of 2.7 therapeutic units per plasmapheresis. The detailed characterization of CCP donors and products has been reported elsewhere (34). The allocation of CCP to a recipient was based on the following criteria, provided availability: ABO-identical units, all 3 CCP units for a patient from 1 donor. If availability of CCP did not allow transfusion of ABO-identical plasma, minor compatible units were also used, i.e., donor plasma did not contain isoagglutinins directed against $\mathrm{ABO}$ antigen(s) present on the recipient's red blood cells, e.g., plasma from a type $\mathrm{AB}$ donor transfused to a type $\mathrm{A}$ recipient. When all criteria were met, readily available products with the highest PRNT50 titers were chosen.

The administration of CCP should commence within 1 day after randomization. One transfusion unit each of CCP was given on days 1, 3, and 5. Since the total amount of neutralizing antibodies depends on both the volume and the antibody titer of CCP, we used "neutralizing units" to take into account both variables. One neutralizing unit was arbitrarily defined as $1 \mathrm{~mL}$ of CCP with a PRNT50 titer of 1:20. The neutralizing units of a CCP transfusion unit were then calculated by dividing the titer by 20 and multiplying by volume (milliliters). In the CCP subgroup that had received a low cumulative amount of neutralizing antibodies, the median titer of transfused plasma units was 1:80 and the mean titer was 1:115. In the CCP subgroup that had received a high cumulative amount of neutralizing antibodies, the median titer of transfused CCP units was 1:320 and the mean titer was 1:396 (Supplemental Figure 1B).

Patients in the crossover group also received 1 unit of CCP on 3 days (day 15,17 , and 19 ). 
Patients in both groups received other antiviral treatment and/or supportive treatment according to institutional standard procedures.

Outcome measures. The primary outcome of the CAPSID trial (to be interpreted as "treatment success") was assessed on day 21 after randomization and is a dichotomous composite outcome of survival and no longer requiring ventilation support or ICU treatment and no tachypnea (i.e., respiratory rate $<30$ breaths per minute) on day 21 . Key secondary outcomes were the time to clinical improvement and the frequency and severity of adverse events (AEs). The key secondary outcome time to clinical improvement was defined as an increase by at least 2 points on the ordinal severity scale (35). Patients without documented improvement were censored at last follow-up. The scale was defined as follows: 0 , no clinical or virological evidence of infection; 1 , ambulatory without limitation of activities; 2 , ambulatory with limitation of activities; 3 , hospitalized without oxygen therapy; 4 , hospitalized with supplemental oxygen by mask or nasal prongs; 5 , hospitalized, noninvasive ventilation or high-flow oxygen; 6, hospitalized, intubation and mechanical ventilation; 7, hospitalized, ventilation and additional organ support (vasopressors, renal replacement therapy, or ECMO); 8, death. Further secondary outcomes were mortality, duration of ventilation support, time to discharge from ICU, time to hospital discharge, and time until negative SARS-CoV-2 PCR from a nasopharyngeal swab. Case fatality rate at days 21,35 , and 60 was calculated by the number of dead patients divided by the total number of cases at the corresponding point in time. Survival time was time from randomization to death in days. Patients not known to have died were censored at last follow-up. Duration of ventilation support was the sum of a patient's single duration of ventilation support and/or ECMO episodes. An episode is defined as the first documentation of a need for noninvasive ventilation or invasive ventilation until the next documentation indicating that no ventilation support (mechanical ventilation, continuous positive airway pressure ventilation, high-flow oxygen, noninvasive ventilation) is needed anymore. Supplemental oxygen (by mask/ nasal prongs) in the weaning period was not considered as ventilation support for this analysis. Length of stay in ICU was defined as interval from the date of randomization to the first date of discharge from the ICU in days. Patients for whom no discharge from ICU was documented were censored at last follow-up. Patients who never entered the ICU were not included in the analysis of the endpoint discharge from ICU. Length of stay in hospital was defined as interval from the date of randomization to the date of hospital discharge (or death) in days. Patients for whom no hospital discharge was documented were censored at last contact. Time until negative SARS-CoV-2 PCR was defined as interval from randomization to first negative SARS-CoV-2 PCR. Patients not known to have a negative result were censored at last follow-up. Frequency of AEs was defined as a patient's total number of documented AEs. Severity of AEs is given as the NCI-Common Terminology Criteria for Adverse Events grade. To determine a patient's worst outcome of an $\mathrm{AE}$, outcomes were ranked as follows: "resolved," "ongoing/resolving," "resolved with sequelae," "unknown," "missing," and "death."

Statistics. All patients were considered for the intention-to-treat (ITT) analysis independently of how they actually conducted the trial until assessment of the primary outcome (day 21).

Nominal and ordinal variables were analyzed by means of absolute frequencies and percentages. Missing values were considered as a separate category. Continuous variables are described by presentation of the median and interquartile range (IQR) for the total number of patients who contributed values. For PRNT50, the geometric mean and variance and $95 \%$ confidence intervals (CIs) are presented. The primary outcome was analyzed using Fisher's exact test comparing the treatment success rates in both treatment groups. A 2-sided $P$ value of less than 0.05 is considered statistically significant. Since the primary outcome is the only confirmatory outcome for this study, an adjustment of the type 1 error due to multiple testing is not required. All other $P$ values are fully explorative. Secondary outcomes were analyzed using a Kaplan-Meier estimation approach. Patients who died during observation without reaching the secondary outcome were censored as if they had reached the end of observation to account for the competing risk setting. Predefined subgroup analyses compared outcome measures in patients with low or high amounts of neutralizing units transfused (cumulative neutralizing units of all transfused CCP products equal to or below the median or above the median) and in subgroups with low or high inflammation. Baseline values of C-reactive protein (CRP), IL-6, and ferritin at baseline were compared with their respective median within the total ITT population, and patients were allocated by the following rules: (a) at least 2 non-missing values of inflammation markers below or equal to the median: "low inflammation markers"; (b) at least 2 non-missing values above the median: "high inflammation markers"; (c) only 2 of the 3 inflammation markers available and 1 below and 1 above the median: "intermediate inflammation markers"; (d) all values missing: "missing." The descriptive statistics of baseline laboratory values are presented in Table 2 . The proportion of patients in the CCP group and the control group with concentrations of the inflammation markers ferritin, CRP, and IL- 6 above the median ("high") or equal to and below the median ("low") is summarized in Supplemental Table 10.

Post hoc analyses were added to compare outcome in patients with presence or absence of neutralizing antibodies at baseline and patients with or without invasive ventilation at baseline.

No imputation was necessary for primary outcome. Missing data for secondary outcomes and AEs were not imputed. All statistical analyses were performed according to the statistical analysis plan using SAS (version 9.4M6 or newer; www.sas.com). The analysis for this article is based on an interim data cutoff on April 28, 2021.

Study approval. The trial was approved by the Federal Authority Paul-Ehrlich-Institute (Langen, Germany) and by the ethics committee of the University of Ulm and the ethics committees of the participating hospitals. The trial is registered at EudraCT (2020-001310-38) and ClinicalTrials.gov (NCT04433910). Written informed consent was obtained from all study participants or their legal representatives.

\section{Author contributions}

HS and SK wrote study protocol, coordinated the study, analyzed and interpreted data, and wrote the manuscript. HS, SK, and ES applied for funding. HS and ES were the lead investigators. MW, DZ, TW, KZ, BG, LE, PS, PML, MB, SZ, GP, JK, MMD, PR, TK, $\mathrm{TT}, \mathrm{HW}, \mathrm{JMK}$, and BJ performed patient care and data collection. VMC and CD performed analysis of SARS-CoV-2 antibodies. MS performed SARS-CoV-2 PCR. TA managed the project. BM performed statistical analysis. All authors approved the manuscript.

\section{Acknowledgments}

We are grateful to the members of the Data Safety Monitoring Board (Jan Beyersmann, Christian von Heymann, Rainer Seitz, 
Carl Kirchmaier) for their advice, and to Philipp Schnecko, Christine Windemuth-Kieselbach, Martin Graeßner, and Gerlinde Schmidtke-Schrezenmeier for expert advice. We thank all the patients who participated in this trial. We thank the clinical research teams, physicians, study nurses, and data managers in all clinical trial centers and the team of the CRO Alcedis. Conducting a clinical trial during a pandemic is a challenge. The commitment to take on substantial extra work when health care resources are already fully occupied by daily care for the severely sick patients deserves special recognition.
The clinical trial CAPSID was supported by the German Federal Ministry of Health (Bundesministerium für Gesundheit). See Supplemental Acknowledgments for details on the CAPSID Clinical Trial Group.

Address correspondence to: Hubert Schrezenmeier, Institute for Clinical Transfusion Medicine and Immunogenetics Ulm, German Red Cross Blood Transfusion Service Baden-WürttembergHessen and University Hospital Ulm and Institute of Transfusion Medicine, University of Ulm, Ulm, Germany. Email: h.schrezenmeier@blutspende.de.
1. Casadevall A, Pirofski L. The convalescent sera option for containing COVID-19. J Clin Invest. 2020;130(4):1545-1548.

2. Bloch EM, et al. Deployment of convalescent plasma for the prevention and treatment of COVID-19. J Clin Invest. 2020;130(6):2757-2765.

3. Joyner MJ, et al. Convalescent plasma antibody levels and the risk of death from Covid-19. N Engl JMed. 2021;384(11):1015-1027.

4. US Food and Drug Administration. FDA Issues Emergency Use Authorization for Convalescent Plasma as Potential Promising COVID-19 Treatment, Another Achievement in Administration's Fight Against Pandemic. https://www.fda.gov/ news-events/press-announcements/fda-issues-emergency-use-authorization-convalescent-plasma-potential-promising-covid-19-treatment. Updated August 23, 2020. Accessed August 31, 2021.

5. Estcourt LJ, Roberts DJ. Convalescent plasma for Covid-19. BMJ. 2020;370:m3516.

6. Li L, et al. Effect of convalescent plasma therapy on time to clinical improvement in patients with severe and life-threatening COVID-19: a randomized clinical trial. JAMA. 2020;4(324):460-470.

7. Agarwal A, et al. Convalescent plasma in the management of moderate covid-19 in adults in India: open label phase II multicentre randomised controlled trial (PLACID Trial). BMJ. 2020;371:m3939.

8. Avendano-Sola C, et al. Convalescent plasma for COVID-19: a multicenter, randomized clinical trial [preprint]. https://doi.org/10.1101/2020.08.26.20 182444. Posted on medRxiv September 29, 2020.

9. Gharbharan A, et al. Effects of potent neutralizing antibodies from convalescent plasma in patients hospitalized for severe SARS-CoV-2 infection. Nat Commun. 2021;12(1):3189.

10. Ray Y, et al. Clinical and immunological benefits of convalescent plasma therapy in severe COVID-19: insights from a single center open label randomised control trial [preprint]. https:// doi.org/10.1101/2020.11.25.20237883. Posted on medRxiv November 29, 2020.

11. Bajpai M, et al. Efficacy of convalescent plasma therapy compared to fresh frozen plasma in severely ill COVID-19 patients: a pilot randomized controlled trial [preprint]. https://doi.org/10 $.1101 / 2020.10 .25 .20219337$. Posted on medRxiv October 27, 2020.
12. AlQahtani M, et al. Randomized controlled trial of convalescent plasma therapy against standard therapy in patients with severe COVID-19 disease. Sci Rep. 2021;11(1):9927.

13. Simonovich VA, et al. A randomized trial of convalescent plasma in Covid-19 severe pneumonia. N Engl J Med. 2020;384(7):619-629.

14. Libster R, et al. Early high-titer plasma therapy to prevent severe Covid-19 in older adults. NEngl J Med. 2021;384(7):610-618.

15. Balcells ME, et al. Early versus deferred antiSARS-CoV-2 convalescent plasma in patients admitted for COVID-19: a randomized phase II clinical trial. PLoS Med. 2021;18(3):e1003415.

16. RECOVERY Collaborative Group. Convalescent plasma in patients admitted to hospital with COVID-19 (RECOVERY): a randomised controlled, open-label, platform trial. Lancet. 2021;397(10289):2049-2059.

17. O'Donnell MR, et al. A randomized double-blind controlled trial of convalescent plasma in adults with severe COVID-19. J Clin Invest. 2021;131(13):e150646.

18. Janiaud P, et al. Association of convalescent plasma treatment with clinical outcomes in patients with COVID-19: a systematic review and meta-analysis. JAMA. 2021;325(12):1185-1195.

19. Piechotta V, et al. Convalescent plasma or hyperimmune immunoglobulin for people with COVID-19: a living systematic review. Cochrane Database Syst Rev. 2021;5(5):CD013600.

20. Klassen SA, et al. The effect of convalescent plasma therapy on mortality among patients with COVID-19: systematic review and meta-analysis. Mayo Clin Proc. 2021;96(5):1262-1275.

21. Jahrsdorfer B, et al. Characterization of the SARS-CoV-2 neutralization potential of COVID-19-convalescent donors. JImmunol. 2021;206(11):2614-2622.

22. Wouters E, et al. Evaluation of SARS-CoV-2 antibody titers and potency for convalescent plasma donation: a brief commentary. Vox Sang. 2021;116(5):493-496.

23. Natarajan $\mathrm{H}$, et al. Markers of polyfunctional SARS-CoV-2 antibodies in convalescent plasma. mBio. 2021;12(2):e00765-21.

24. Winkler ES, et al. Human neutralizing antibodies against SARS-CoV-2 require intact Fc effector functions for optimal therapeutic protection. Cell. 2021;184(7):1804-1820.
25. Acosta-Ampudia Y, et al. COVID-19 convalescent plasma composition and immunological effects in severe patients. J Autoimmun. 2021;118:102598.

26. Anand SP, et al. Longitudinal analysis of humoral immunity against SARS-CoV-2 Spike in convalescent individuals up to 8 months post-symptom onset. Cell Rep Med. 2021;2(6):100290.

27. Salazar E, et al. Significantly decreased mortality in a large cohort of Coronavirus Disease 2019 (COVID-19) patients transfused early with convalescent plasma containing high-titer anti-severe acute respiratory syndrome Coronavirus 2 (SARS-CoV-2) spike protein IgG. Am J Pathol. 2021;191(1):90-107.

28. Zhou Y, et al. Comorbidities and the risk of severe or fatal outcomes associated with coronavirus disease 2019: a systematic review and metaanalysis. Int J Infect Dis. 2020;99:47-9956.

29. Martin-Vicente M, et al. Absent or insufficient anti-SARS-CoV-2 $\mathrm{S}$ antibodies at ICU admission are associated to higher viral loads in plasma, antigenemia and mortality in COVID19 patients [preprint]. https://doi.org/10.110 1/2021.03.08.21253121. Posted on medRxiv March 08, 2021.

30. Joyner MJ, et al. Safety update: COVID-19 convalescent plasma in 20,000 hospitalized patients. Mayo Clin Proc. 2020;95(9):1888-1897.

31. Lim ZJ, et al. Case fatality rates for patients with COVID-19 requiring invasive mechanical ventilation. A meta-analysis. Am J Respir Crit Care Med. 2021;203(1):54-66.

32. Wölfel R, et al. Virological assessment of hospitalized cases of coronavirus disease 2019. Nature. 2020;581:465-469.

33. Kreye J, et al. A therapeutic non-self-reactive SARS-CoV-2 antibody protects from lung pathology in a COVID-19 hamster model. Cell. 2020;183(4):1058-1069.

34. Körper S, et al. Donors for SARS-CoV-2 convalescent plasma for a controlled clinical trial: donor characteristics, content and time course of SARS-CoV-2 neutralizing antibodies. Transfus Med Hemother. 2021;48(3):137-147.

35. World Health Organization. COVID-19 Therapeutic Trial Synopsis. https://www.who. int/publications/i/item/covid-19-therapeutic-trial-synopsis. Updated February 18, 2020. Accessed August 31, 2021. 